\title{
Pretreatment Heterogeneous
} Enhancement Pattern of Hepatocellular Carcinoma May Be a Useful New Predictor of Early Response to Lenvatinib and Overall Prognosis

\author{
Yusuke Kawamura $^{a, c}$ Masahiro Kobayashi ${ }^{a, c}$ Junichi Shindoh ${ }^{\text {b, c }}$ \\ Yuta Kobayashib, c Kayoko Kasuyaa, c Tomoya Sano ${ }^{a, c}$ \\ Shunichiro Fujiyama ${ }^{a, c}$ Tetsuya Hosakaa, c Satoshi Saitoha, c \\ Hitomi Sezaki ${ }^{a, c}$ Norio Akuta ${ }^{a, c}$ Fumitaka Suzuki ${ }^{a, c}$ \\ Yoshiyuki Suzuki ${ }^{a, c}$ Kenji Ikedaa, ${ }^{a}$ Yasuji Arase ${ }^{a, c}$ Masaji Hashimotob, c \\ Hiromitsu Kumada ${ }^{a}$ c \\ ${ }^{a}$ Department of Hepatology, Toranomon Hospital, Tokyo, Japan; ${ }^{b}$ Hepatobiliary-Pancreatic \\ Surgery Division, Department of Gastroenterological Surgery, Toranomon Hospital, Tokyo, \\ Japan; ' Okinaka Memorial Institute for Medical Research, Toranomon Hospital, Tokyo, \\ Japan
}

\section{Keywords}

Computed tomography · Hepatocellular carcinoma - Lenvatinib · Malignant potential ·

Poorly-differentiated hepatocellular carcinoma

\begin{abstract}
Objective: The aim of this study was to evaluate the performance of pretreatment computed tomography (CT) enhancement of hepatocellular carcinoma (HCC) as a potential predictor of response to lenvatinib and its relevance to survival outcomes. Methods: We evaluated 51 consecutive patients who received lenvatinib treatment for unresectable HCC. On imaging analysis, pretreatment arterial/portal phase dynamic CT images were classified as follows: type 2, homogeneous enhancement pattern with increased arterial blood flow; type 3, heterogeneous enhancement pattern with a septum-like structure; and type 4, heterogeneous enhancement pattern with irregularly shaped ring structures. Treatment response was evaluated using modified Response Evaluation Criteria in Solid Tumors at 2-12 weeks after initiation of lenvatinib, and the correlations between the CT enhancement patterns and response to lenvatinib or survival outcomes were investigated. Results: Of the 51 patients, 38 (75\%) experi-
\end{abstract}


enced an objective response (OR). ORs were significantly more common in heterogeneously enhanced HCC (types 3 and 4) than in homogeneous HCC (type 2) (83 vs. 53\%, respectively; $p=0.037)$. Multivariate analysis revealed that pretreatment heterogeneous enhancement pattern is an independent predictor for response to lenvatinib (odds ratio, 4.75; $p=0.042$ ). Presence of OR was associated with longer progression-free survival (PFS) (hazard ratio, 0.36; $p=$ 0.017 ), and patients with oncologically aggressive type 3 and 4 tumors showed similar PFS to those harboring type 2 tumors ( $p=0.455$ ), reflecting that OR was more common in type 3 or 4 tumors compared with type 2 tumors. Although postprogression survival was extremely poor in patients with type 4 tumors $(p=0.064)$, overall survival after introduction of lenvatinib was not statistically different among the three groups of patients $(p=0.053)$. Conclusion: The $\mathrm{CT}$ enhancement pattern of HCC may predict response to lenvatinib. OR seems to occur more frequently in HCC with oncologically aggressive features and may contribute to prolonged survival through a prolonged progression-free interval, even in an oncologically poor-risk group of patients.

(C) 2020 The Author(s)

Published by S. Karger AG, Basel

\section{Introduction}

Hepatocellular carcinoma (HCC) is the most common type of liver cancer, which is the third leading cause of cancer [1]. For HCC, the Barcelona Clinic Liver Cancer (BCLC) staging system is currently most widely used [2,3]. BCLC intermediate-stage disease may be further subclassified based on the Up-to-7 criteria [4] and liver function with the Child-Pugh system [5]. In patients with tumors within the Up-to-7 criteria and with good liver function, transarterial chemoembolization (TACE) is recommended. Previously, many chemotherapeutic agents were used for chemoembolization, and TACE was repeatedly performed until treatment could no longer be administered. Although response may improve if different agents are used (especially cisplatin $[6,7]$ ), the efficacy of TACE is limited. As a result of repeated TACE, many patients become TACE-refractory, and liver function declines [8]. About 10 years ago, the molecular targeted agent sorafenib became available for the treatment of unresectable HCC $[9,10]$, and worldwide, HCC treatment trended toward switching from TACE to sorafenib before liver function decline [11-14]. The OPTIMIS study [14] also suggested that switching from intraarterial treatment to sorafenib could extend survival. However, the efficacy of sorafenib in this setting is modest: the median overall survival (OS) is $<1$ year and the objective response rate (ORR) is $<5 \%$. Thus, there remains a critical and unmet need for aggressive development of new and more effective agents for advanced HCC.

Recently, prior to approval elsewhere in the world, lenvatinib became available as a new molecular targeted agent for the first-line treatment of unresectable advanced HCC in Japan [15]. Lenvatinib, an inhibitor of vascular endothelial growth factor receptor 1-3, fibroblast growth factor receptor 1-4 (FGFR1-4), platelet-derived growth factor receptor alpha, Ret, and Kit, was reported to be noninferior to sorafenib with respect to OS in patients with untreated advanced HCC $[16,17]$. It was the second molecular targeted agent to demonstrate efficacy as a first-line treatment for unresectable HCC; lenvatinib treatment results in a higher ORR compared with sorafenib treatment (18.8 vs. 6.5\%, respectively, as evaluated by Response Evaluation Criteria in Solid Tumors version 1.1 [RECIST 1.1]) [16].

On the other hand, in several treatment algorithms [2,3], only the presence or absence of tumor hypervascularity on contrast enhancement studies is recommended for optimizing the selection of treatment for HCC, without evaluation of the malignant potential of the target nodules. We previously reported that a "heterogeneous enhancement pattern with a septumlike structure" in the arterial phase of dynamic computed tomography (CT) analysis accu-

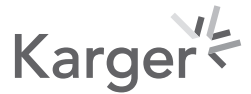


rately predicts macroscopic classification of the nodular types [18] of simple nodular type with extranodular growth (SNEG) and confluent multinodular (CMN) type of HCC, and that a "heterogeneous enhancement pattern with irregular ring-like structures" in the arterial phase of dynamic CT accurately predicts the histopathological grade of poorly-differentiated HCCs; we named these enhancement patterns type 3 and type 4, respectively [19].

These unique enhancement patterns are also correlated with the oncological aggressiveness of HCC [20], and they may guide an optimal approach for ablation therapy [21]. Given the potential ability of the enhancement patterns of HCC in predicting the malignant potential of tumors and responses to treatment, we sought to investigate the powers of these unique radiological enhancement patterns as a new pretreatment surrogate marker for predicting the response to lenvatinib and overall prognosis.

\section{Patients and Methods}

\section{Study Population}

From April 2018 to September 2019, 87 patients received systemic anticancer treatment using lenvatinib for unresectable HCC at the Department of Hepatology, Toranomon Hospital, Tokyo, Japan. In this study, the following inclusion criteria were used: (1) triple-phase dynamic CT study performed within 1 month prior to initiation of lenvatinib, (2) tumor with hyperenhancement in the arterial phase of dynamic CT, (3) performance of triple-phase dynamic CT to evaluate treatment response 2-12 weeks after initiation of lenvatinib, (4) Child-Pugh class A liver function at the time of lenvatinib initiation, (5) BCLC stage A to C tumor(s), (6) unresectable HCC and patient does not want to undergo local ablation or chemoembolization therapy for various reasons (i.e., tumor size, number and location, extrahepatic metastasis, TACE refractoriness, and various complications), (7) no treatment history of lenvatinib, (8) at least one measurable target nodule in the liver, (9) treatment interval of $>28$ days since previous tyrosine kinase inhibitor (TKI; sorafenib or regorafenib) therapy, and (10) an observation period of $\geq 4$ weeks. Eventually, 51 patients met the inclusion criteria.

\section{Contrast Infusion and CT Protocol}

All patients underwent triple-phase dynamic CT. CT was performed with a 64-multidetector CT scanner (Aquilion 64; Canon Medical Systems, Tochigi, Japan) with the following scanning parameters: rotation time $0.5 \mathrm{~s}$, beam collimation $64 \times 0.5 \mathrm{~mm}$, section thickness and interval $5 \mathrm{~mm}$, beam pitch 0.83 , tube voltage $120 \mathrm{kV}$, and tube current $150 \mathrm{mAs}$. All helical scans were started at the top of the liver and proceeded in a cephalocaudal direction. Unenhanced and three-phase contrast-enhanced helical scans of the whole liver were acquired. Patients were instructed to hold their breath with exhalation during scanning. An automatic bolus-tracking program (Sure Start; Canon Medical Systems) was used to time the start of acquisition in each phase after contrast injection (nonionic contrast material with a concentration of $350 \mathrm{mg}$ iodine/mL iomeprol [Iomeron 350; Eisai, Tokyo, Japan] at a dose of 100-120 mL/body). Attenuation at the axis of the celiac artery level was monitored by one radiology technician; the region of interest (ROI) cursor $\left(1 \mathrm{~cm}^{2}\right)$ was placed in the abdominal aorta. Real-time low-dose $(120 \mathrm{kV}, 25 \mathrm{mAs})$ serial monitoring studies were begun $5 \mathrm{~s}$ after the start of contrast injection. The trigger threshold level was set at 100 Hounsfield units (HU). Double arterial phase acquisition was started 15 and $20 \mathrm{~s}$ after triggering, and portal phase and delayed phase acquisition were started 70 and $180 \mathrm{~s}$ after the start of the contrast injection, respectively.

\section{Diagnosis of HCC}

The diagnosis of HCC was based predominantly on image analysis using dynamic CT. When a liver nodule showed hyperattenuation in the arterial phase of the dynamic study and washout in the portal or delayed phase, the nodule was diagnosed as HCC.

\section{Imaging Analysis of HCC and Definitions of Dynamic CT Enhancement Patterns}

Before treatment, the dynamic study enhancement pattern on the arterial and portal phases was classified into one of four types defined in our previous report [19]. The type 1 pattern represented a homogeneous enhancement pattern with no increase in arterial blood flow, and the entire image was uniform during the arterial and portal phases. The type 2 pattern represented a homogeneous enhancement pattern with 


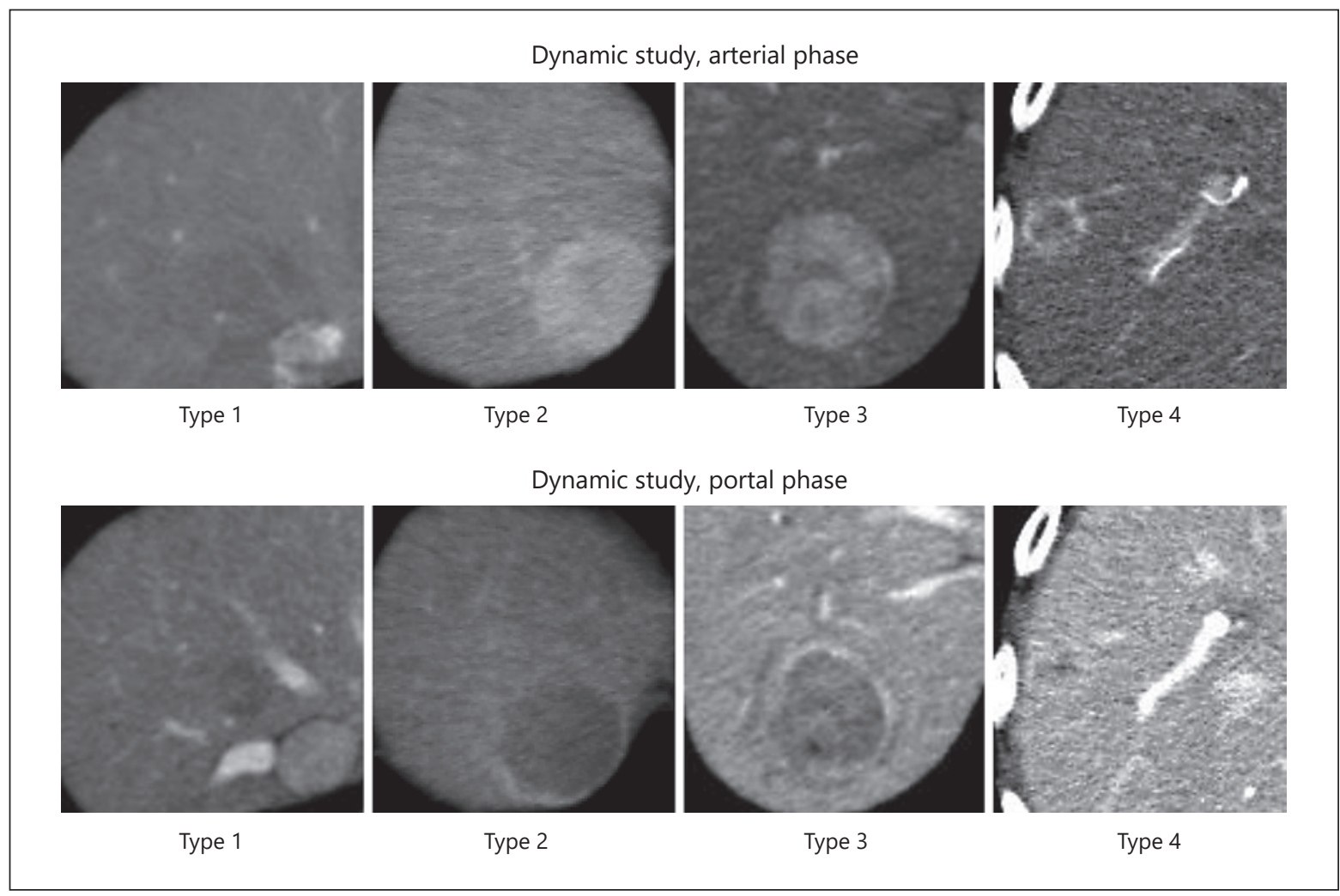

Fig. 1. Original dynamic study images with each of the four enhancement patterns. Reprinted with permission from John Wiley and Sons [20].

increased arterial blood flow, and the entire image was uniform during the arterial and portal phases. The type 3 pattern represented a heterogeneous enhancement pattern with septations, with heterogeneous enhancement and septations in the arterial phase, while the septations resembled a near-uniform tumor tissue periphery in the portal phase. The type 4 pattern represented a heterogeneous enhancement pattern with irregular ring-like structures; the arterial phase was marked by the presence of irregularly shaped ring areas of enhancement and areas of little blood flow relative to the periphery of the tumor tissue, and the portal phase was characterized by areas of reduced blood flow (Fig. 1).

The enhancement pattern on the arterial and portal phases of dynamic CT was assessed independently by an expert hepatologist (Y. Kawamura) and an expert hepatobiliary surgeon (J. Shindoh) who were blinded to the clinical data. Discrepancies between these two examiners were resolved by consensus review including an additional reviewer (K. Ikeda). Generally, macroscopic classification of the nodular type of SNEG and CMN types strongly relates to the type 3 enhancement pattern, and histologically, the type 1 enhancement pattern represents well-differentiated HCC, while the type 2 and 3 patterns represent moderately-differentiated HCC; the type 4 enhancement pattern is a significantly specific feature for predicting poorly-differentiated HCC [19].

In this study, all target HCC nodules appeared to be hypervascular; therefore, we classified all nodules into three enhancement patterns (types 2 to 4 ). The enhancement pattern that accounts for $70 \%$ of the nodule is defined as the predominant enhancement pattern.

In addition, we analyzed the mean HU of intrahepatic target tumors; a circular ROI was drawn on the axial plane to include the largest surface of the target lesion, and the mean HU of each tumor was calculated [22].

\section{Lenvatinib Treatment and Adverse Event Assessment}

Lenvatinib (Eisai, Tokyo, Japan) was given orally to the majority of patients at either $8 \mathrm{mg} /$ day for patients $<60 \mathrm{~kg}$ or $12 \mathrm{mg} /$ day for patients $\geq 60 \mathrm{~kg}$; treatment was discontinued when any unacceptable or serious adverse events (AEs) or significantly clinical tumor progression were observed. According to the 
guidelines for administration of lenvatinib, the drug dose should be reduced or the treatment interrupted when a patient develops grade $\geq 3$ severe AEs or any unacceptable grade 2 drug-related AEs occur. AEs were assessed using the National Cancer Institute's Common Terminology Criteria for Adverse Events, version 4.0 [23]. If a drug-related $\mathrm{AE}$ occurred, dose reduction or temporary interruption was maintained until the symptom was resolved to grade 1 or 2 , according to the guidelines provided by the manufacturer.

\section{Treatment Response Evaluation}

Treatment response was evaluated in accordance with modified RECIST (mRECIST) [24], and RECIST 1.1 [25] was used as an auxiliary. We assessed the best tumor response during 2-12 weeks. The liver was examined by dynamic CT.

Treatment response was assessed independently by an expert hepatologist (Y. Kawamura) and an expert hepatobiliary surgeon (J. Shindoh) who were blinded to the clinical data. Discrepancies between these two examiners were resolved by consensus review including an additional reviewer (K. Ikeda).

\section{Definition of TACE Failure/Refractoriness}

TACE failure was defined as an insufficient response after $\geq 2$ consecutive TACE procedures as evident on response evaluation CT or magnetic resonance imaging after 1-3 months, even after the chemotherapeutic agent had been changed and/or the feeding artery had been reanalyzed. In addition, appearance of a higher number of lesions in the liver than that recorded at the previous TACE procedure (other than the nodule being treated) was added to the definition of TACE failure/refractoriness [26].

\section{Assessment of Hepatic Functional Reserve}

Child-Pugh classification [5] and albumin-bilirubin (ALBI) grade [27] were used to assess hepatic functional reserve. Modified ALBI (mALBI) grade was based on the ALBI score, which was calculated from serum albumin and total bilirubin concentrations using the following formula: ALBI score $=\left[\log _{10}\right.$ bilirubin ( $\mu \mathrm{mol} /$ $\mathrm{L}) \times 0.66]+$ [albumin $(\mathrm{g} / \mathrm{L}) \times-0.085]$, and defined by the following cutoffs: $\leq-2.60=$ grade $1,>-2.60$ to $\leq-2.27$ = grade $2 \mathrm{a},>-2.27$ to $\leq-1.39=$ grade $2 \mathrm{~b},>-1.39=$ grade 3 [28].

\section{Follow-Up Protocol}

Physicians examined patients every 1-2 weeks after initiation of lenvatinib, and biochemical laboratory and urine tests were also performed. After initiation of lenvatinib, patients underwent dynamic CT to evaluate early treatment response at 2-12 weeks. After the first evaluation of treatment response, dynamic CT was performed every 2-3 months.

\section{Statistical Analysis}

Statistical analysis was performed using IBM SPSS software ver. 26.0 (SPSS Inc., USA). Data are expressed as median and range. Differences in background features between each parameter were analyzed by $\chi^{2}$ test, Fisher exact test, Mann-Whitney U test, and Kruskal-Wallis test. $p$ values $<0.05$ were considered to indicate statistical significance. The progression-free survival (PFS), postprogression survival (PPS), and OS after the introduction of lenvatinib were estimated with the Kaplan-Meier method of comparing values with a log-rank test.

To identify factors associated with objective response (OR) after initiation of lenvatinib, a multivariate analysis was performed using logistic regression with backward elimination. Among potential independent variables, factors with a marginal association $(p<0.2)$ in the univariate analysis were included in the initial model. Then, after stepwise selection, only factors that showed a statistically significant association with OR at $p<0.1$ were included in the final model. Predictive factors for PFS were also investigated with the Cox proportional hazards model with a similar variable selection method.

\section{Results}

\section{Clinical Profiles and Laboratory Data}

Table 1 summarizes the clinical profile and laboratory data of 51 HCC patients treated with lenvatinib in this study. The male:female ratio was 2.19:1. Hepatitis $\mathrm{C}$ virus antibody was detected in $54.9 \%$ of patients. Overall, 47 patients (92\%) received an initial dose of lenvatinib 
Table 1. Clinical profiles and laboratory data of patients with HCC treated with lenvatinib

Patient characteristics and laboratory data

Patients

Male:female sex

Age, years

Body mass index

Body weight $<60 \mathrm{~kg}: \geq 60 \mathrm{~kg}$

HCV:HBV:NonB,NonC

Performance status 0:1

Platelet count, $\times 10^{3} / \mu \mathrm{L}$

Albumin, g/dL

Total bilirubin, mg/dL

Prothrombin activity, \%

AST, IU/L

AFP, $\mu \mathrm{g} / \mathrm{L}$

DCP, AU/L

Child-Pugh score 5:6

mALBI score 1:2a:2b:3

Initial dose of lenvatinib, $4 \mathrm{mg}: 8 \mathrm{mg}: 12 \mathrm{mg}$

Reduced starting dose of lenvatinib

History of TKI treatment

Tumor characteristics

Tumor diameter, $\mathrm{mm}$

Number of tumors

Macrovascular invasion

Extrahepatic metastasis

BCLC stage A:B:C

TACE failure/refractoriness

Pretreatment dynamic CT study enhancement pattern

Type 2 /type 3 /type 4
51

$35: 16$

$74(45-91)$

$22.3(11.9-30.1)$

$32: 19$

28:6:17

$48(94 \%): 3(6 \%)$

$122(48-280)$

$3.7(3.0-4.5)$

$1.0(0.3-2.8)$

$82.8(64.9-124.8)$

$40(15-351)$

$189(0.8-61,040.7)$

$277(13-63,347)$

$30(59 \%): 21(41 \%)$

10 (20\%):20 (39\%):21 (41\%):0 (0\%)

2 (4\%):28 (55\%):21 (41\%)

$4(8 \%)$

$4(8 \%)$

$31.8(11.0-112.7)$

$4(1-200)$

$9(18 \%)$

$18(35 \%)$

5 (10\%):23 (45\%):23 (45\%)

$41(80 \%)$

$15(29 \%) / 24(47 \%) / 12(24 \%)$

Values are presented as $n, n(\%)$, or median (range). AFP, alpha-fetoprotein; AST, aspartate aminotransferase; BCLC, Barcelona Clinic Liver Cancer; CT, computed tomography; DCP, des-gamma-carboxy prothrombin; HBV, hepatitis B virus; HCC, hepatocellular carcinoma; HCV, hepatitis C virus; mALBI, modified albumin-bilirubin; NonB,NonC, neither HBV nor HCV infection present; TACE, transarterial chemoembolization; TKI, tyrosine kinase inhibitor.

according to body weight, and 4 patients $(8 \%)$ received a reduced starting dose for the following reasons: age $>80$ years, platelet count $<50 \times 10^{3} / \mu \mathrm{L}$, and body mass index $<19$. In addition, 4 patients (8\%) received a higher starting dose of lenvatinib according to body weight because they were enrolled in a global phase II study with fixed dosing (12 mg). With regard to liver function, 30 (59\%) patients presented with a Child-Pugh score of 5, and 10 patients $(20 \%)$ presented with an mALBI grade of 1 . Based on pretreatment image analysis, the median tumor diameter was $31.8 \mathrm{~mm}$, and 23 of 51 patients (45\%) presented with BCLC stage $\mathrm{C}$ disease; 9 of these 23 patients (39\%) presented with macrovascular invasion (Vp2, $n=6 ; \mathrm{Vp} 3, n=1 ; \mathrm{Vp} 3$ and $\mathrm{Vv} 3, n=1 ; \mathrm{Vp} 4, n=2)$, and 18 of 23 patients (78\%) presented with extrahepatic metastasis. In addition, 4 patients (8\%) had a history of treatment with other TKIs, and 41 patients ( $80 \%)$ had a TACE failure/refractoriness status. The median number (range) of TACE treatments was $3(0-20)$ before initiation of lenvatinib. The median levels of alpha-fetoprotein (AFP) and des-gamma-carboxy prothrombin (DCP) were $189 \mu \mathrm{g} / \mathrm{L}$ and 277 $\mathrm{AU} / \mathrm{L}$, respectively. The median (range) relative dose intensity (RDI) of lenvatinib was $100 \%$ $(40-150 \%)$ at 2 weeks, $92 \%(32-150 \%)$ at 4 weeks, $74 \%(30-150 \%)$ at 8 weeks, and $68 \%$ (31-138\%) at 12 weeks.

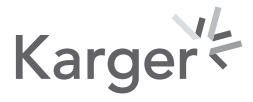




\begin{tabular}{l|l}
\hline Liver Cancer 2020;9:275-292 \\
\hline DOI: 10.1159/000505190 & $\begin{array}{l}\text { @ 2020 The Author(s). Published by S. Karger AG, Basel } \\
\text { www.karger.com/lic }\end{array}$ \\
\hline
\end{tabular}

Table 2. Early treatment response evaluation using mRECIST 4-12 weeks after initiation of lenvatinib in all patients and by BCLC stage

\begin{tabular}{|c|c|c|c|c|c|c|c|}
\hline Category & TACE failure/ & Macrovascular & Extrahepatic & \multicolumn{4}{|c|}{ Response evaluation using mRECIST } \\
\hline $\begin{array}{l}\text { All cases } \\
(n=51)\end{array}$ & $41(80 \%)$ & $9(18 \%)$ & $18(35 \%)$ & $\begin{array}{l}\text { CR } \\
6(12 \%) \\
\text { OR } \\
38(75 \%)\end{array}$ & $\begin{array}{l}\text { PR } \\
32(63 \%)\end{array}$ & $\begin{array}{l}\text { SD } \\
9(18 \%)\end{array}$ & $\begin{array}{l}\text { PD } \\
4(8 \%)\end{array}$ \\
\hline $\begin{array}{l}\text { BCLC stage A/B } \\
(n=28)\end{array}$ & $24(86 \%)$ & $0(0 \%)$ & $0(0 \%)$ & $\begin{array}{l}\text { CR } \\
4(14 \%) \\
\text { OR } \\
18(64 \%)\end{array}$ & $\begin{array}{l}\text { PR } \\
14(50 \%)\end{array}$ & $\begin{array}{l}\text { SD } \\
7(25 \%)\end{array}$ & $\begin{array}{l}\text { PD } \\
3(11 \%)\end{array}$ \\
\hline $\begin{array}{l}\text { BCLC stage C } \\
(n=23)\end{array}$ & 17 (74\%) & $9(39 \%)$ & $18(78 \%)$ & $\begin{array}{l}\text { CR } \\
2(9 \%) \\
\text { OR } \\
20(87 \%)\end{array}$ & $\begin{array}{l}\text { PR } \\
18(78 \%)\end{array}$ & $\begin{array}{l}\text { SD } \\
2(9 \%)\end{array}$ & $\begin{array}{l}\text { PD } \\
1(4 \%)\end{array}$ \\
\hline
\end{tabular}

Values are presented as $n(\%)$. The composition ratio is rounded off to the first decimal place and therefore the total will not necessarily be 100. BCLC, Barcelona Clinic Liver Cancer; CR, complete response; mRECIST, modified Response Evaluation Criteria in Solid Tumors; OR, objective response; PD, progressive disease; PR, partial response; SD, stable disease; TACE, transarterial chemoembolization.

With respect to the pretreatment dynamic CT enhancement pattern, 15 patients (29\%) had the type 2 pattern, 24 patients ( $47 \%)$ had the type 3 pattern, and 12 patients $(24 \%)$ had the type 4 pattern. Fifteen patients had died at the time of database lock (November 5, 2019); the median duration of lenvatinib administration was 6.7 months, and the median observation period was 10.6 months. The weighted $\kappa$ value of the imaging analysis between the two independent examiners (Y. Kawamura and J. Shindoh) was 0.972.

\section{Evaluation of Treatment Response to Lenvatinib}

In early treatment response evaluation using mRECIST, 6 of 51 patients (12\%) experienced a complete response (CR), 32 (63\%) experienced a partial response (PR), 9 (18\%) had stable disease, and 4 (8\%) had progressive disease (PD); therefore, 38 of 51 patients $(75 \%)$ experienced an OR. By BCLC stage (A or B vs. C), the ORR was $64 \%$ for patients with BCLC stage $A$ or $B$ disease and $87 \%$ for patients with BCLC stage $C$ disease (Table 2). TACE failure/ refractoriness was present in 24 of 28 patients (86\%) with BCLC stage A/B disease and 17 of 23 (74\%) with BCLC stage C disease. Among patients with BCLC stage C disease, 18 of 23 (78\%) had extrahepatic spread; however, an interventional treatment approach was enforced for an extrahepatic metastatic lesion in only 1 patient (6\%).

By mALBI grade ( 1 vs. 2 a vs. 2 b), the ORR was $80 \%$ ( 8 of 10 patients) for grade $1,80 \%$ (16 of 20 patients) for grade $2 \mathrm{a}$, and $67 \%$ (14 of 21 patients) for grade $2 \mathrm{~b}$; there were no significant differences in ORR among grades $(p=0.561)$.

\section{Evaluation of Treatment Response after Initiation of Lenvatinib by Dynamic CT}

Enhancement Pattern (Types 2 to 4)

In the early treatment response evaluation based on the dynamic CT enhancement pattern by mRECIST, the ORR of each enhancement pattern (types 2, 3, and 4) was 53, 79, and $92 \%$, respectively. The ORR was significantly higher with the heterogeneous than with the homogeneous enhancement pattern (83 vs. 53\%, respectively) ( $p=0.037$ ) (Table 3).

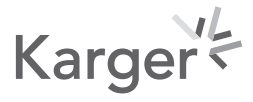


Table 3. Evaluation of early treatment response after initiation of lenvatinib by dynamic CT enhancement pattern (types 2 to 4 ) and analysis of imaging features using mRECIST

\begin{tabular}{|c|c|c|c|c|c|}
\hline \multirow[b]{2}{*}{$\begin{array}{l}\text { Homogeneous } \\
\text { enhancement } \\
\text { pattern }\end{array}$} & \multirow{2}{*}{$\begin{array}{l}\text { Dynamic CT image of } \\
\text { main target nodule } \\
\text { type } 2(n=15)\end{array}$} & \multicolumn{4}{|c|}{ Response evaluation using mRECIST, $n(\%)$} \\
\hline & & $\begin{array}{l}\text { CR } \\
1(7 \%) \\
\text { ORR } \\
53 \%\end{array}$ & $\begin{array}{l}\text { PR } \\
7(47 \%)\end{array}$ & $\begin{array}{l}\text { SD } \\
6(40 \%)\end{array}$ & $\begin{array}{l}\text { PD } \\
1(7 \%)\end{array}$ \\
\hline \multirow[t]{2}{*}{$\begin{array}{l}\text { Heterogeneous } \\
\text { enhancement } \\
\text { pattern }\end{array}$} & type $3(n=24)$ & $\begin{array}{l}\text { CR } \\
3(13 \%) \\
\text { ORR } \\
79 \%\end{array}$ & $\begin{array}{l}\text { PR } \\
16(67 \%)\end{array}$ & $\begin{array}{l}\text { SD } \\
3(13 \%)\end{array}$ & $\begin{array}{l}\text { PD } \\
2(8 \%)\end{array}$ \\
\hline & type $4(n=12)$ & $\begin{array}{l}\text { CR } \\
2(17 \%) \\
\text { ORR } \\
92 \%\end{array}$ & $\begin{array}{l}\text { PR } \\
9(75 \%)\end{array}$ & $\begin{array}{l}\text { SD } \\
0(0 \%)\end{array}$ & $\begin{array}{l}\text { PD } \\
1(8 \%)\end{array}$ \\
\hline
\end{tabular}

The composition ratio is rounded off to the first decimal place and therefore the total will not necessarily be 100. CR, complete response; CT, computed tomography; mRECIST, modified Response Evaluation Criteria in Solid Tumors; ORR, objective response rate; PD, progressive disease; PR, partial response; SD, stable disease.

The weighted $\kappa$ value of treatment response evaluation using mRECIST between the two independent examiners (Y. Kawamura and J. Shindoh) was 0.902.

On the other hand, in the early treatment response evaluation based on the dynamic CT enhancement pattern by RECIST 1.1, the ORR of each enhancement pattern (types 2, 3, and 4) was 40,54 , and $58 \%$, respectively. There were no significant differences between heterogeneous and homogeneous enhancement pattern ( 56 vs. $40 \%$, respectively) ( $p=0.311$ ) (Table 4).

However, no patient had an mRECIST evaluation of CR or PR, in spite of RECIST 1.1 PD.

\section{Rate of Decrease in ROI after Initiation of Lenvatinib}

We compared the rate of decrease in the ROI of target tumors after initiation of lenvatinib with therapeutic effect using mRECIST. The ROI after the initiation of lenvatinib was measured at the time of image evaluation.

According to the tumor enhancement patterns (homogeneous vs. heterogeneous), the ROI showed a significant decrease after the initiation of lenvatinib (median rate of change in ROI: homogeneous enhancement pattern, $-17.95 \%$; heterogeneous enhancement pattern, $-31.28 \% ; p=0.033$ ) (Fig. 2a).

On the other hand, according to tumor enhancement patterns (types 2 to 4), the ROI showed a tendency to decrease (median rate of change in ROI: type 2 enhancement pattern, $-17.95 \%$; type 3 enhancement pattern, $-32.46 \%$; type 4 enhancement pattern, $-28.25 \%$; $p=$ 0.099) (Fig. 2b).

Finally, according to early treatment response evaluation using mRECIST (non-OR vs. OR), the ROI showed a significant decrease between before and after the initiation of lenvatinib (median rate of change in ROI: non-OR, $-12.17 \%$; OR, $-30.63 \%$; $p=0.014$ ) (Fig. 2c).

\section{Predictors of OR to Lenvatinib}

Among the 19 potential independent variables listed in Table 5, serum albumin level (g/ dL) $(p=0.110)$, serum aspartate aminotransferase (AST) level (IU/L) $(p=0.178)$, serum AFP 


\begin{tabular}{|c|c|}
\hline \multicolumn{2}{|l|}{ Liver Cancer 2020;9:275-292 } \\
\hline DOI: 10.1159/000505190 & $\begin{array}{l}\text { (c) } 2020 \text { The Author(s). Published by S. Karger AG, Basel } \\
\text { www.karger.com/lic }\end{array}$ \\
\hline
\end{tabular}

Table 4. Evaluation of early treatment response after initiation of lenvatinib by dynamic CT enhancement pattern (types 2 to 4 ) and analysis of imaging features using RECIST 1.1

\begin{tabular}{|c|c|c|c|c|c|}
\hline \multirow[b]{2}{*}{$\begin{array}{l}\text { Homogeneous } \\
\text { enhancement } \\
\text { pattern }\end{array}$} & \multirow{2}{*}{$\begin{array}{l}\text { Dynamic CT image of } \\
\text { main target nodule } \\
\text { type } 2(n=15)\end{array}$} & \multicolumn{4}{|c|}{ Response evaluation using RECIST, $n(\%)$} \\
\hline & & $\begin{array}{l}\text { CR } \\
0(0 \%) \\
\text { ORR } \\
40 \%\end{array}$ & $\begin{array}{l}\text { PR } \\
6(40 \%)\end{array}$ & $\begin{array}{l}\text { SD } \\
8(53 \%)\end{array}$ & $\begin{array}{l}\text { PD } \\
1(7 \%)\end{array}$ \\
\hline \multirow[t]{2}{*}{$\begin{array}{l}\text { Heterogeneous } \\
\text { enhancement } \\
\text { pattern }\end{array}$} & type $3(n=24)$ & $\begin{array}{l}\text { CR } \\
0(0 \%) \\
\text { ORR } \\
54 \%\end{array}$ & $\begin{array}{l}\text { PR } \\
13(54 \%)\end{array}$ & $\begin{array}{l}\text { SD } \\
9(38 \%)\end{array}$ & $\begin{array}{l}\text { PD } \\
2(8 \%)\end{array}$ \\
\hline & type $4(n=12)$ & $\begin{array}{l}\text { CR } \\
0(0 \%) \\
\text { ORR } \\
58 \%\end{array}$ & $\begin{array}{l}\text { PR } \\
7(58 \%)\end{array}$ & $\begin{array}{l}\text { SD } \\
4(33 \%)\end{array}$ & $\begin{array}{l}\text { PD } \\
1(8 \%)\end{array}$ \\
\hline
\end{tabular}

The composition ratio is rounded off to the first decimal place and therefore the total will not necessarily be 100. CR, complete response; CT, computed tomography; RECIST, Response Evaluation Criteria in Solid Tumors; ORR, objective response rate; PD, progressive disease; PR, partial response; SD, stable disease.

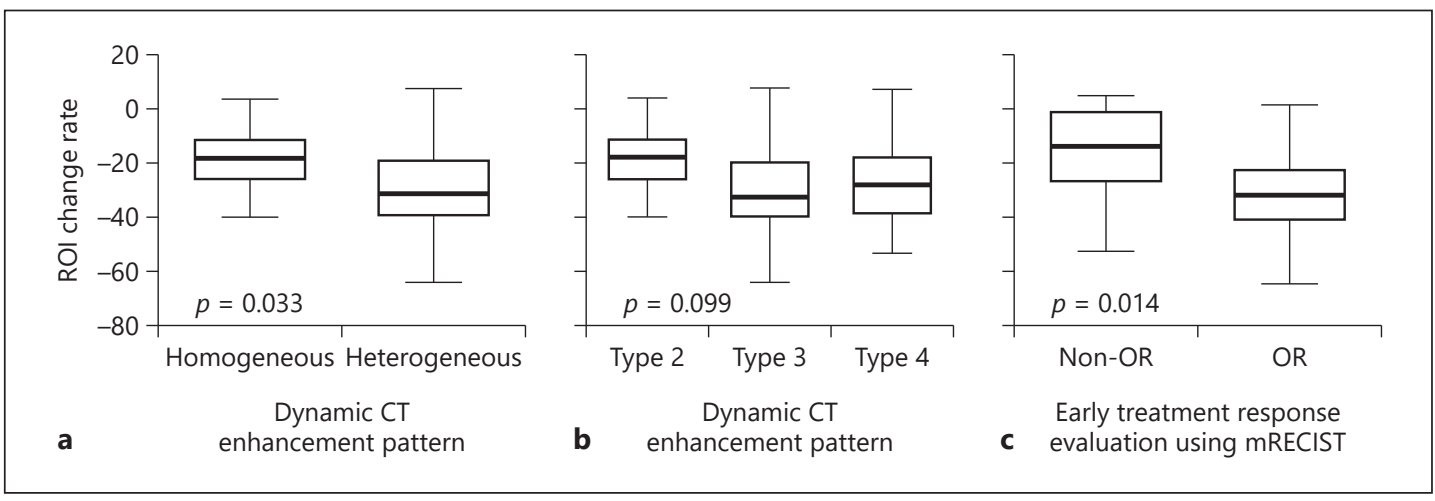

Fig. 2. ROI rate of change before and after lenvatinib administration, according to dynamic CT enhancement pattern and early treatment responses. a Homogeneous vs. heterogeneous. b Tumor enhancement patterns. c Non-OR vs. OR. CT, computed tomography; mRECIST, modified Response Evaluation Criteria in Solid Tumors; OR, objective response; ROI, region of interest.

level $(\mu \mathrm{g} / \mathrm{L})(p=0.168)$, plasma DCP level (AU/L) ( $p=0.121)$, extrahepatic metastases ( $p=$ 0.037 ), and heterogeneous enhancement pattern (types 3 and 4) on pretreatment dynamic CT $\operatorname{scan}(p=0.031)$ were included in the multivariate logistic regression model, and only a heterogeneous CT enhancement pattern was selected as an independent predictor for OR after the introduction of lenvatinib (odds ratio, 4.75; 95\% confidence interval, 1.06-21.25; $p=0.042$ ).

\section{Predictors of PFS after Introduction of Lenvatinib}

Of the 20 variables tested for their potential to predict PFS (Table 6), body mass index $(p=0.188)$, serum AST level (IU/L) $(p=0.15)$, serum AFP level $(\mu \mathrm{g} / \mathrm{L})(p=0.004)$, plasma DCP level (AU/L) $(p=0.029)$, tumor diameter $(\mathrm{cm})(p=0.025)$, macrovascular invasion $(p=$ 
Table 5. Predictive factors for early treatment response to lenvatinib

\begin{tabular}{lllllll}
\hline & $p^{1}$ & Coefficients $^{2}$ & SE & Wald $\chi^{2}$ & $\begin{array}{l}\text { Odds } \\
\text { ratio }\end{array}$ & $95 \%$ CI \\
\hline $\begin{array}{l}\text { Heterogeneous dynamic CT } \\
\text { enhancement pattern prior to } \\
\text { introduction of lenvatinib }\end{array}$ & 0.042 & 1.558 & 0.764 & 4.2 & 4.75 & $1.06-21.25$ \\
\hline \begin{tabular}{l} 
Extrahepatic metastases \\
\hline
\end{tabular} & 0.084 & 1.942 & 1.125 & 3.0 & 6.97 & $0.77-63.20$ \\
\hline
\end{tabular}

CI, confidence interval; CT, computed tomography; SE, standard error. ${ }^{1}$ Based on likelihood test adjusted for the other factors in the final model. ${ }^{2}$ Estimated coefficient for the variable and the associated SE. Multivariate logistic regression was applied with stepwise backward selection. Among potential predictors, factors presenting marginal association $(p<0.2)$ with objective response to lenvatinib in univariate analysis were included in the initial model. Then factors that showed no or limited statistically significant association $(p>0.1)$ adjusted for the remaining factors in the model were deleted from the model in stepwise fashion. The 19 tested variables were as follows ( $p$ values in univariate analysis): age (0.712), sex (0.525), body mass index (0.993), body weight ( $<60 \mathrm{~kg}$ vs. $\geq 60 \mathrm{~kg}$ ) (0.576), etiology (hepatitis C virus vs. others) (0.929), serum albumin (0.110), serum total bilirubin (0.399), prothrombin activity (0.735), platelet count (0.634), serum aspartate aminotransferase (0.178), serum alpha-fetoprotein (0.168), des-gamma-carboxy prothrombin (0.121), tumor diameter (0.248), tumor number ( $>4$ vs. $\leq 4)(0.957)$, macrovascular invasion (0.554), extrahepatic metastasis (0.037), heterogeneous CT enhancement pattern prior to treatment (0.031), transarterial chemoembolization failure/refractoriness (0.705), and reduced starting dose of lenvatinib (0.262).

Table 6. Predictive factors for PFS after introduction of lenvatinib

\begin{tabular}{lllllll}
\hline & $p^{1}$ & Coefficients $^{2}$ & SE & Wald $\chi^{2}$ & HR & $95 \%$ CI \\
\hline AFP $>100 \mu \mathrm{g} / \mathrm{L}$ & 0.016 & 0.007 & 0.003 & 5.8 & 1.01 & $1.00-1.01$ \\
\hline OR at 2-12 weeks & 0.017 & -1.019 & 0.426 & 5.7 & 0.36 & $0.16-0.83$ \\
\hline $\begin{array}{l}\text { TACE failure/ } \\
\text { refractoriness }\end{array}$ & 0.028 & -0.994 & 0.451 & 4.9 & 0.37 & $0.15-0.90$ \\
\hline
\end{tabular}

AFP, alpha-fetoprotein; CI, confidence interval; HR, hazard ratio; OR, objective response; PFS, progressionfree survival; SE, standard error; TACE, transarterial chemoembolization. ${ }^{1}$ Based on likelihood test adjusted for the other factors in the final model. ${ }^{2}$ Estimated coefficient for the variable and the associated SE. Multivariate Cox regression was applied with stepwise backward selection. Among potential predictors, factors presenting marginal association $(p<0.2)$ with PFS after introduction of lenvatinib in univariate analysis were included in the initial model. Then factors that showed no or limited statistically significant association $(p>$ 0.1 ) adjusted for the remaining factors in the model were deleted from the model in stepwise fashion. The 20 tested variables were as follows ( $p$ values in univariate analysis): age (0.386), sex (0.794), body mass index (0.188), body weight ( $<60 \mathrm{~kg}$ vs. $>60 \mathrm{~kg}$ ) (0.313), etiology (hepatitis C virus vs. others) (0.402), serum albumin (0.221), serum total bilirubin (0.240), prothrombin activity (0.632), platelet count (0.619), serum aspartate aminotransferase (0.100), serum AFP (0.004), plasma des-gamma-carboxy prothrombin (0.029), tumor diameter (0.025), tumor number ( $>4$ vs. $\leq 4)(0.622)$, macrovascular invasion $(0.016)$, extrahepatic metastasis (0.520), heterogeneous computed tomography enhancement pattern prior to treatment (0.602), TACE failure/refractoriness (0.011), reduced starting dose of lenvatinib (0.515), and OR at 2-12 weeks (0.011). 


\section{Liver Cancer}

Fig. 3. Adjusted PFS curves according to presence of OR to lenvatinib. HR, hazard ratio; OR, objective response; PFS, progression-free survival.

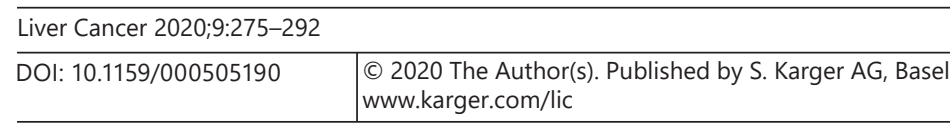

Kawamura et al.: Lenvatinib for Unresectable Hepatocellular Carcinoma

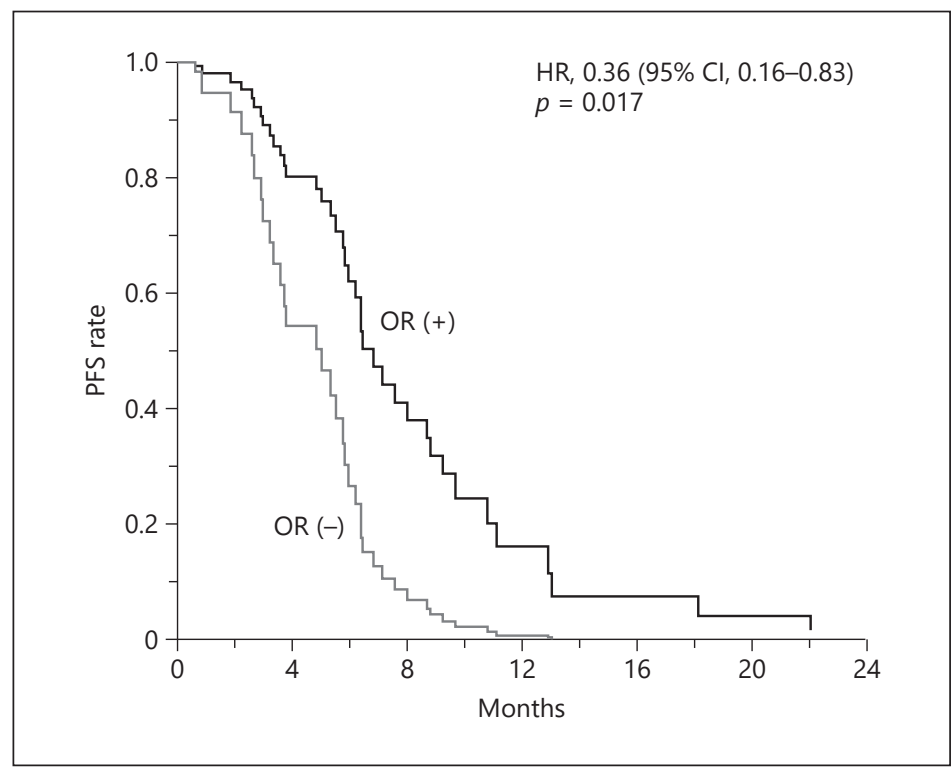

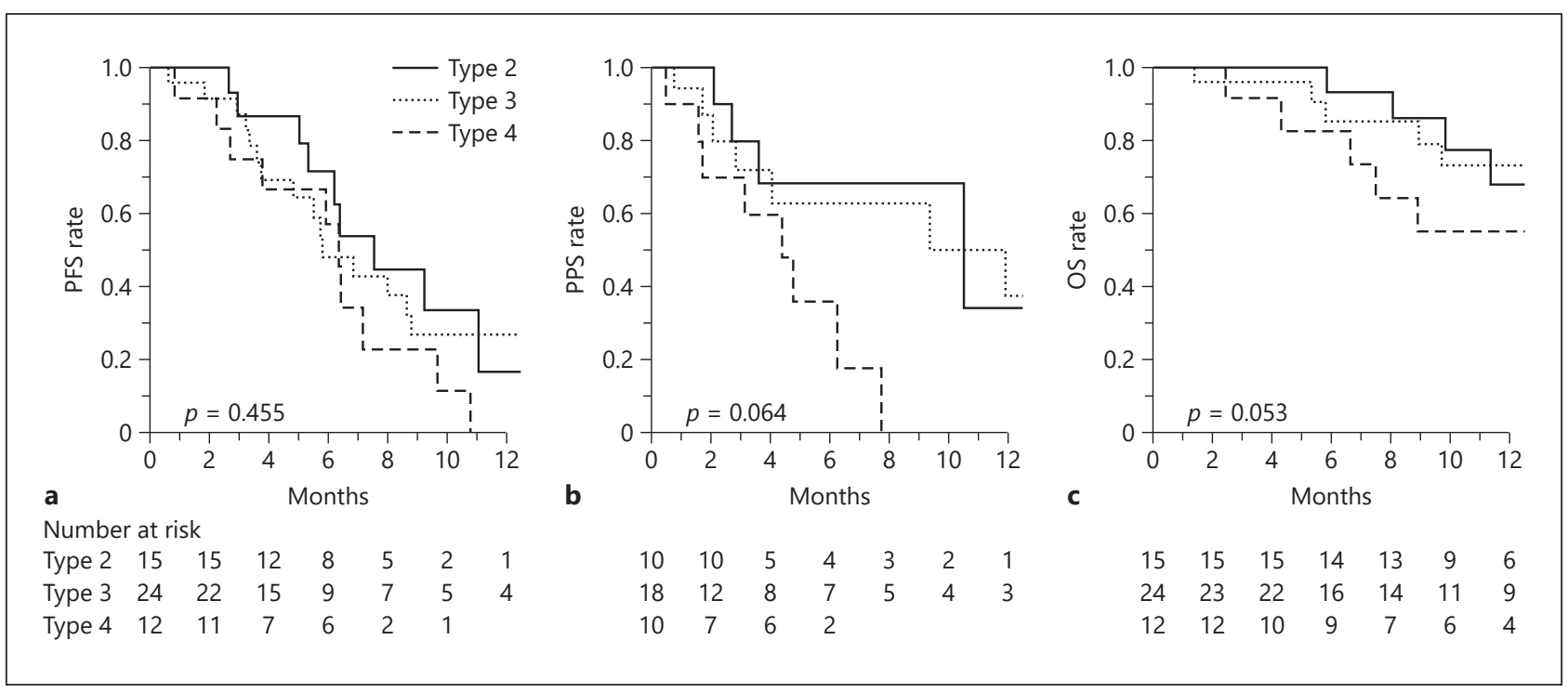

Fig. 4. Survival outcomes according to the patterns of tumor enhancement on CT scan prior to the introduction of lenvatinib. a PFS rate. b PPS rate. c OS rate. CT, computed tomography; OS, overall survival; PFS, progression-free survival; PPS, postprogression survival.

0.016), TACE failure/refractoriness ( $p=0.011$ ), and OR at 2-12 weeks after the introduction of lenvatinib $(p=0.011)$ were included in the initial Cox regression model. Stepwise variable selection identified serum AFP level $>100 \mu \mathrm{g} / \mathrm{L}$ (hazard ratio, 1.01; 95\% confidence interval, $1.00-1.01 ; p=0.016$ ), OR at $2-12$ weeks after the introduction of lenvatinib (hazard ratio, 0.36 ; $95 \%$ confidence interval, $0.16-0.83 ; p=0.017$ ), and TACE failure/refractoriness (hazard ratio, $0.37 ; 95 \%$ confidence interval, $0.15-0.90 ; p=0.028$ ) as independent predictors of PFS. Adjusted PFS curves showed clear differences according to the presence of OR to lenvatinib (Fig. 3). 
PPS in Lenvatinib-Treated Patients according to Pretreatment Dynamic CT Patterns

Thirty-eight patients were diagnosed with PD during the observation period; 21 of 38 patients (55\%) continued to receive lenvatinib treatment, and 8 of these 21 patients $(38 \%)$ received TACE sequential therapy.

Figure 4 shows survival outcomes according to the CT enhancement pattern. Although there was no difference in PFS among the three groups, PPS was markedly worse when a patient presented with a type 4 enhancement pattern before the introduction of lenvatinib (Fig. 4a, b). As a result, cumulative survival after the introduction of lenvatinib was not significantly different among the three groups (Fig. 4c).

Frequency of Grade $\geq 3$ AEs following Initiation of Lenvatinib

No grade 4 AEs were reported during the observation period. With respect to grade 3 AEs, 6 of 51 patients (12\%) experienced hypertension, 4 of $51(8 \%)$ experienced a hand-foot skin reaction, 3 of $51(6 \%)$ experienced appetite loss, 2 of $51(4 \%)$ experienced diarrhea, 9 of 51 (18\%) experienced decreased platelet counts, 2 of 51 (4\%) experienced elevated AST, 5 of $51(10 \%)$ experienced elevated total bilirubin, 5 of 51 (10\%) experienced hepatic encephalopathy, and 2 of 51 (4\%) experienced elevated urinary protein.

\section{Discussion}

Patients with unresectable HCC who show intolerability to sorafenib or failure of another TKI need second- and third-line treatment options. Lenvatinib, a newer TKI $[15,16]$, has become available as a first-line agent for unresectable HCC in Japan. Lenvatinib demonstrated efficacy as a first-line treatment for unresectable HCC and is associated with a higher ORR compared with sorafenib [16].

Notably, in patients with advanced HCC, we frequently encounter several tumors and various enhancement patterns within a single patient's liver. Therefore, it is difficult to select the best overall treatment method and chemotherapeutic agent by sampling just one of many tumors. Moreover, tumor biopsies are not easy to perform in all cases for several reasons, including tumor localization, risk of tumor dissemination, and risk of bleeding due to liver cirrhosis.

In these situations, we previously reported that a type 3 enhancement pattern accurately predicts macroscopic classification of the nodular type of SNEG and CMN types of HCC and that a type 4 enhancement pattern accurately predicts the histopathological grade of poorlydifferentiated HCC [19]. Our past work revealed a strong relationship between type 4 enhancement pattern and HCC recurrence characterized by multiple nodules and/or portal vein invasion following radiofrequency ablation [20].

However, the utility of these dynamic CT enhancement patterns in predicting responses to TKIs, especially lenvatinib, has not been clear until now. In this study, a heterogeneous enhancement pattern (types 3 and 4) on dynamic CT was a significant pretreatment predictor of OR by mRECIST following initiation of lenvatinib. In contrast, with RECIST 1.1, there were no significant differences between enhancement pattern and treatment response. However, in additional analyses, (1) no patient had an mRECIST evaluation of CR or PR, in spite of RECIST 1.1 PD, (2) the ROIs of the target tumors were significantly decreased in patients who had an OR compared with patients who did not, and (3) the ROIs of the target tumors decreased more in those with heterogeneous enhancement patterns, including the type 4 enhancement pattern (Fig. 2a-c). From these results, we concluded that there was no overestimation for decreased blood flow in tumors with heterogeneous enhancement patterns using mRECIST evaluation.

\section{Karger's}


Several years ago, Harimoto et al. [29] reported that high FGFR2 expression is significantly correlated with poor histological differentiation, a higher incidence of portal vein invasion, and high AFP levels. In contrast to sorafenib and regorafenib, lenvatinib inhibits FGFR1-4 in addition to vascular endothelial growth factor receptor 1-3, platelet-derived growth factor receptor alpha, Ret, and Kit. The role of FGFR inhibition by lenvatinib may have influenced the results of the present study. However, many points regarding the details of this mechanism are unclear. On the other hand, although there was no difference in PFS among the three groups, in the survival analysis PPS was significantly worse when a patient presented with a type 4 enhancement pattern before the introduction of lenvatinib. As a result, cumulative survival after the introduction of lenvatinib was not significantly different among the three groups.

The importance of this result can also be seen from previous clinical research: ${ }^{18} \mathrm{~F}$-fluorodeoxyglucose positron emission tomography/CT positivity was reported to be strongly associated with poorly-differentiated HCC [30]. In such a clinical situation, significantly lower PFS and OS in sorafenib-treated, positron emission tomography-positive HCC was reported [31]. From this report, in general, poorly-differentiated HCC has an extremely poor prognosis. Compared with this previous clinical report, it may be considered that lenvatinib showed sufficient clinical benefit for PFS and OS in HCC of high malignant potential in this study.

Recently, a relationship between a sustained decrease in AFP from 2 to 4 weeks after initiation of lenvatinib and achievement of a highly OR was reported [32]. To investigate this point, we performed an additional analysis. To align with the previously reported study, we excluded 13 patients with normal AFP $(<20 \mu \mathrm{g} / \mathrm{L}$; 9 of these 13 patients [69\%] presented with a heterogeneous enhancement pattern), and we analyzed 38 patients with abnormal AFP (24 patients had a sustained decrease in AFP and 14 patients did not). In our cohort, 16 of the 24 cases $(67 \%)$ with a sustained decrease in AFP showed an OR, and 12 of the 14 cases (86\%) without a sustained decrease in AFP showed an OR; there was no significant difference in response rate between groups $(p=0.269)$. With regard to the enhancement pattern, 27 patients had a heterogeneous enhancement pattern, and 17 of the 27 (63\%) showed a sustained decrease in AFP, while the remaining 11 patients had a homogeneous enhancement pattern, and 7 of the $11(64 \%)$ showed a sustained decrease in AFP. Finally, in patients with sustained decreases in AFP, 13 of 17 (76\%) with a heterogeneous enhancement pattern had an OR, and 3 of 7 (43\%) with a homogeneous enhancement pattern had an OR. On the other hand, all 10 patients with a heterogeneous enhancement pattern who did not have a sustained decrease in AFP had an OR (100\%), and 2 of 4 with a homogeneous enhancement pattern who did not have a sustained decrease in AFP had an OR (50\%). However, both the previous report [32] and our study contained a small number of cases, so further study is necessary.

By BCLC clinical stage, the ORR was $64 \%$ among patients with stage A or B disease, while it was $87 \%$ in patients with stage $C$ disease. An explanation for this result is that in this cohort, the rate of intrahepatic target nodules that presented with a heterogeneous enhancement pattern (types 3 and 4 ) was $61 \%$ in the BCLC stage A/B group and $83 \%$ in the BCLC stage C group. Furthermore, the rate of intrahepatic target nodules that presented with a type 4 enhancement pattern was significantly higher in the BCLC stage C group (39\%) compared with the BCLC stage A/B group (11\%) ( $p=0.023)$.

In this study, nodules with a heterogeneous enhancement pattern, especially a type 4 enhancement pattern, showed significantly higher early treatment response rates compared with nodules with a homogeneous enhancement pattern $(p=0.042)$. Therefore, the high distribution of target nodules with heterogeneous enhancement in this cohort may have influenced the relatively high response rate in this cohort. In addition, 41 of 51 patients $(80 \%)$ presented TACE failure/refractoriness, and the high number of TACE treatments (median of 3) before initiation of lenvatinib may have caused the high distribution of heterogeneously

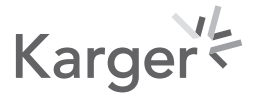


Fig. 5. Concept of therapeutic effectiveness considering tumor differentiation. The rate of tumor growth decreased (downward arrows), and PFS was extended (rightward arrows), regardless of tumor differentiation. However, poorly-differentiated HCC has poor PPS; therefore, we predict that the prognosis of poorly-differentiated HCC will not be better than that of moderately-differentiated HCC. HCC, hepatocellular carcinoma; PFS, progression-free survival; PPS, postprogression survival.

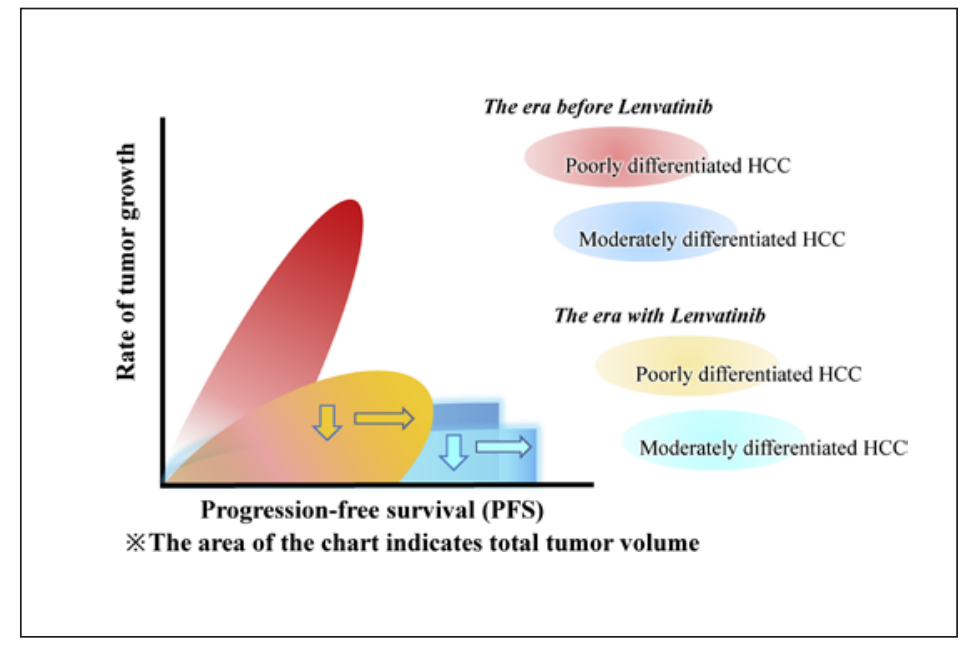

enhanced target nodules in this cohort. Previously, other researchers reported that TACE treatment induced a more malignant phenotype of HCC, including poorly-differentiated histology and CK19 expression indicative of the more aggressive biliary phenotype [33, 34].

In multivariate analysis, TACE failure/refractoriness was identified as one of the positive predictive factor for PFS in this study. The high number of TACE treatments before initiation of lenvatinib may also have strongly influenced this result.

On the other hand, in this study cohort, 30 of 51 patients (59\%) received dynamic CT two to four times for evaluation of an early treatment response 2-12 weeks after lenvatinib initiation, and the median RDI of lenvatinib was $100 \%$ at 2 weeks, $92 \%$ at 4 weeks, $74 \%$ at 8 weeks, and $68 \%$ at 12 weeks. The RDI was kept at a relatively high level during those $2-12$ weeks as a result of close follow-up every 1-2 weeks and early AE management. Therefore, it is possible that many cases received dynamic $\mathrm{CT}$ at the best time for evaluation of treatment response. In addition, in this study the mALBI score had no effect on early treatment response. During the high-RDI period, the compatibility of tumor features and lenvatinib may most strongly affect early treatment response.

We suggest that as a result of these background features, this study cohort showed a relatively high response rate compared with the results of the previously reported global phase III REFLECT trial [16].

Figure 5 illustrates our concept of therapeutic effectiveness considering tumor differentiation.

In this study, HCC nodules with a heterogeneous enhancement pattern showed a significant early tumor response; however, there was no significant difference in PFS according to the heterogeneity of the dynamic CT enhancement pattern. Moreover, in the multivariate analysis, OR at 2-12 weeks was an independent predictive factor for favorable PFS. We speculated that even if HCC is of high malignant potential (type 3 and 4 enhancement patterns), if an OR is achieved, it does not affect PFS. On the other hand, in the type 4 enhancement group, the PPS was extremely poor, and no patient survived 9 months after being diagnosed with PD.

From these results, lenvatinib decreases the rate of tumor growth and extends PFS, regardless of tumor differentiation. However, the estimated PPS of poorly-differentiated HCC is extremely poor; therefore, we predict that the prognosis of poorly-differentiated HCC will not exceed that of moderately-differentiated HCC.

In this study, we performed a multivariate analysis to evaluate predictive factors for PFS, including treatment response at 2-12 weeks. In general, we should perform these 
Fig. 6. Strategy of lenvatinib administration for patients with HCC tumors that are unresectable and have failed or are refractory to TACE. CT, computed tomography; HCC, hepatocellular carcinoma; mRECIST, modified Response Evaluation Criteria in Solid Tumors; TACE, transarterial chemoembolization.

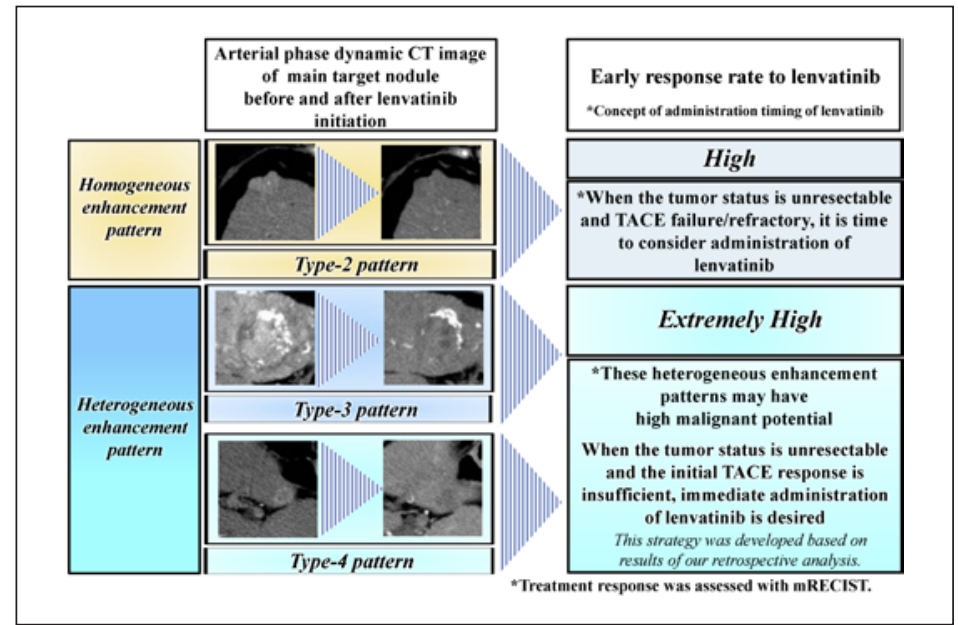

analyses using only pretreatment data. However, as is well known, lenvatinib achieves an extremely rapid treatment response, and this rapid response reflects PFS. In addition, adjusted PFS curves showed a clear difference according to the presence of OR to lenvatinib (Fig. 3).

The ability to predict the prognosis more accurately at the early stage of treatment is extremely useful in clinical practice. Therefore, in this study, we carried out a multivariate analysis including the effects of early treatment response at 2-12 weeks to identify predictive factors for PFS. However, an insufficient amount of preclinical or clinical data regarding relationship estimation of tumor differentiation using dynamic CT enhancement pattern and treatment response to TKIs exists. These potential mechanisms need to be further investigated in future studies.

Figure 6 illustrates our proposed treatment strategy. For tumors with a homogeneous enhancement pattern, the early response rate to lenvatinib was high (ORR, 53\%). Therefore, for tumors that are unresectable and have failed or are refractory to TACE, administration of lenvatinib should be considered. In contrast, tumors with a heterogeneous enhancement pattern (types 3 and 4) associated with a malignant gross type (SNEG and CMN) and that are histologically poorly differentiated [19] demonstrated a poor response to TACE [18, 35]. However, their response to lenvatinib was extremely high (ORR, 79-92\%). Therefore, when the tumor status is unresectable and the initial TACE response is insufficient, lenvatinib should be immediately administered. However, since this strategy was developed based on the results of our small retrospective analysis, a multicenter study enrolling a larger number of patients is required to verify this strategy.

The majority of cases examined in this study are postmarketing cases, so long-term analysis has not yet been completed. However, after lenvatinib administration had been initiated in 1 patient (BCLC stage C) in a global phase III trial and in 3 patients (1 BCLC stage $\mathrm{B}$ and $2 \mathrm{BCLC}$ stage $\mathrm{C}$ ) in the postmarketing period, a cancer-free state was achieved following additional treatment. In addition, 2 of these patients have remained cancer-free even after discontinuing lenvatinib. Compared with other TKIs, lenvatinib appears to decrease blood flow very rapidly, and as a result, evaluation of response to the agent can be done quickly. In both of the cases described above, lenvatinib played a role as a bridging therapy for surgical resection, with the aim of achieving a cancer-free state. Most importantly, no new lesions appeared within the treatment period. Even if new lesions appear after surgical resection, they could possibly be controlled following reinitiation of lenvatinib. 
This study has some limitations. First, this was a retrospective, single-center, cohort study that evaluated a small number of patients. Second, the follow-up period of this trial was short compared with that of the global phase III REFLECT trial [16] (median follow-up period of 10.6 vs. 27.7 months); therefore, it is not yet possible to perform a high-quality prognostic analysis. Third, in this study, we evaluated the treatment response at 2-12 weeks. It is possible that the length of the evaluation period has some influence on the result. Finally, the diagnosis of HCC was based essentially on image analysis. A large-scale study is required to evaluate the utility of heterogeneous dynamic study enhancement patterns as biomarkers in the treatment of HCC by lenvatinib.

\section{Conclusion}

The CT enhancement pattern of HCC may predict response to lenvatinib. OR seems to occur more frequently in HCC presenting with oncologically aggressive features and may contribute to prolonged survival through a prolonged progression-free interval even in an oncologically poor-risk group of patients.

\section{Acknowledgement}

This work was supported in part by grants from the Ministry of Health, Labor, and Welfare of Japan and the Japan Agency for Medical Research and Development.

\section{Statement of Ethics}

All procedures were in accordance with the ethical standards of the responsible committees on human experimentation (institutional and national) and with the Helsinki Declaration of 1975. The study was approved by the Institutional Review Board of Toranomon Hospital (protocol number 1438-H/B).

\section{Disclosure Statement}

Y. Kawamura, MD, PhD reports honoraria from Eisai. M. Kobayashi, MD reports honoraria from Eisai. J. Shindoh, MD, PhD reports honoraria from Eisai. H. Kumada, MD, PhD reports honoraria from Eisai. The other authors declare no conflicts of interest.

\section{Funding Sources}

Okinaka Memorial Institute for Medical Research and Japanese Ministry of Health, Labor, and Welfare.

\section{Author Contributions}

Y. Kawamura, MD, PhD: study concept and design, acquisition of data, statistical analysis, drafting of the manuscript. M. Kobayashi, MD: acquisition of data, statistical analysis. J. Shindoh, MD, PhD: acquisition of data, statistical analysis, critical revision. Y. Kobayashi, MD: acquisition of data. K. Kasuya, MD: acquisition of data. T. Sano, MD: acquisition of data. S. Fujiyama, MD: acquisition of data. T. Hosaka, MD: acquisition of data. S. Saitoh, MD: acquisition of data. H. Sezaki, MD: acquisition of data. N. Akuta, MD, PhD: acquisition of data. F. Suzuki, MD, PhD: acquisition of data. Y. Suzuki, MD, PhD: acquisition of data. K. Ikeda, MD, PhD: acquisition of data, statistical analysis, study supervision. Y. Arase, MD, PhD: acquisition of data. M. Hashimoto, MD, PhD: acquisition of data. H. Kumada, MD, PhD: acquisition of data. All authors had access to the data and participated in the writing of the manuscript. 


\begin{tabular}{l|l|}
\hline Liver Cancer 2020;9:275-292 \\
\hline DOI: 10.1159/000505190 & $\begin{array}{l}\text { @ 2020 The Author(s). Published by S. Karger AG, Basel } \\
\text { www.karger.com/lic }\end{array}$ \\
\hline
\end{tabular}

\section{References}

1 Ferlay J, Soerjomataram I, Dikshit R, Eser S, Mathers C, Rebelo M, et al. Cancer incidence and mortality worldwide: sources, methods and major patterns in GLOBOCAN 2012. Int J Cancer. 2015 Mar;136(5):E359-86.

2 Forner A, Reig ME, de Lope CR, Bruix J. Current strategy for staging and treatment: the BCLC update and future prospects. Semin Liver Dis. 2010 Feb;30(1):61-74.

3 Forner A, Reig M, Bruix J. Hepatocellular carcinoma. Lancet. 2018 Mar;391(10127):1301-14.

4 Mazzaferro V, Llovet JM, Miceli R, Bhoori S, Schiavo M, Mariani L, et al.; Metroticket Investigator Study Group. Predicting survival after liver transplantation in patients with hepatocellular carcinoma beyond the Milan criteria: a retrospective, exploratory analysis. Lancet Oncol. 2009 Jan;10(1):35-43.

5 Pugh RN, Murray-Lyon IM, Dawson JL, Pietroni MC, Williams R. Transection of the oesophagus for bleeding oesophageal varices. Br J Surg. 1973 Aug;60(8):646-9.

6 Kawamura Y, Ikeda K, Hirakawa M, Hosaka T, Kobayashi M, Saitoh S, et al. Efficacy of platinum analogue for advanced hepatocellular carcinoma unresponsive to transcatheter arterial chemoembolization with epirubicin. Hepatol Res. 2009 Apr;39(4):346-54.

7 Seki A, Hori S. Switching the loaded agent from epirubicin to cisplatin: salvage transcatheter arterial chemoembolization with drug-eluting microspheres for unresectable hepatocellular carcinoma. Cardiovasc Intervent Radiol. 2012 Jun;35(3):555-62.

8 Hiraoka A, Kumada T, Kudo M, Hirooka M, Koizumi Y, Hiasa Y, et al.; Real-life Practice Experts for HCC (RELPEC) Study Group and HCC 48 Group (hepatocellular carcinoma experts from 48 clinics). Hepatic Function during Repeated TACE Procedures and Prognosis after Introducing Sorafenib in Patients with Unresectable Hepatocellular Carcinoma: multicenter Analysis. Dig Dis. 2017;35(6):602-10.

9 Llovet JM, Ricci S, Mazzaferro V, Hilgard P, Gane E, Blanc JF, et al.; SHARP Investigators Study Group. Sorafenib in advanced hepatocellular carcinoma. N Engl J Med. 2008 Jul;359(4):378-90.

10 Cheng AL, Kang YK, Chen Z, Tsao CJ, Qin S, Kim JS, et al. Efficacy and safety of sorafenib in patients in the AsiaPacific region with advanced hepatocellular carcinoma: a phase III randomised, double-blind, placebocontrolled trial. Lancet Oncol. 2009 Jan;10(1):25-34.

11 Ogasawara S, Chiba T, Ooka Y, Kanogawa N, Motoyama T, Suzuki E, et al. Efficacy of sorafenib in intermediatestage hepatocellular carcinoma patients refractory to transarterial chemoembolization. Oncology. 2014; 87(6):330-41.

12 Arizumi T, Ueshima K, Minami T, Kono M, Chishina H, Takita M, et al. Effectiveness of Sorafenib in Patients with Transcatheter Arterial Chemoembolization (TACE) Refractory and Intermediate-Stage Hepatocellular Carcinoma. Liver Cancer. 2015 Dec;4(4):253-62.

13 Ohki T, Kondo M, Karasawa Y, Kawamura S, Maeshima S, Kojima K, et al. Evaluation of the efficacy of sorafenib on overall survival in patients with hepatocellular carcinoma using FT rate: a devised index. Adv Ther. 2017 May;34(5):1097-108.

14 Peck-Radosavljevic M, Kudo M, Raoul JL, Lee HC, Decaens T, Heo J, et al. Outcomes of patients (pts) with hepatocellular carcinoma (HCC) treated with transarterial chemoembolization (TACE): Global OPTIMIS final analysis. J Clin Oncol. 2018;36(suppl):abstr 4018.

15 Ikeda K, Kudo M, Kawazoe S, Osaki Y, Ikeda M, Okusaka T, et al. Phase 2 study of lenvatinib in patients with advanced hepatocellular carcinoma. J Gastroenterol. 2017 Apr;52(4):512-9.

16 Kudo M, Finn RS, Qin S, Han KH, Ikeda K, Piscaglia F, et al. Lenvatinib versus sorafenib in first-line treatment of patients with unresectable hepatocellular carcinoma: a randomised phase 3 non-inferiority trial. Lancet. 2018 Mar;391(10126):1163-73.

17 Matsuki M, Hoshi T, Yamamoto Y, Ikemori-Kawada M, Minoshima Y, Funahashi Y, et al. Lenvatinib inhibits angiogenesis and tumor fibroblast growth factor signaling pathways in human hepatocellular carcinoma models. Cancer Med. 2018 Jun;7(6):2641-53.

18 Kanai T, Hirohashi S, Upton MP, Noguchi M, Kishi K, Makuuchi M, et al. Pathology of small hepatocellular carcinoma. A proposal for a new gross classification. Cancer. 1987 Aug;60(4):810-9.

19 Kawamura Y, Ikeda K, Hirakawa M, Yatsuji H, Sezaki H, Hosaka T, et al. New classification of dynamic computed tomography images predictive of malignant characteristics of hepatocellular carcinoma. Hepatol Res. 2010 Oct; 40(10):1006-14.

20 Kawamura Y, Ikeda K, Seko Y, Hosaka T, Kobayashi M, Saitoh S, et al. Heterogeneous type 4 enhancement of hepatocellular carcinoma on dynamic CT is associated with tumor recurrence after radiofrequency ablation. AJR Am J Roentgenol. 2011 Oct;197(4):W665-73.

21 Kawamura Y, Ikeda K, Fujiyama S, Hosaka T, Kobayashi M, Saitoh S, et al. Potential of a no-touch pincer ablation procedure that uses a multipolar radiofrequency ablation system to prevent intrasubsegmental recurrence of small and single hepatocellular carcinomas. Hepatol Res. 2017 Sep;47(10):1008-20.

22 Ronot M, Bouattour M, Wassermann J, Bruno O, Dreyer C, Larroque B, et al. Alternative Response Criteria (Choi, European Association for the Study of the Liver, and modified Response Evaluation Criteria in Solid Tumors [RECIST]) versus RECIST 1.1 in patients with advanced hepatocellular carcinoma treated with sorafenib. Oncologist. 2014 Apr; 19(4):394-402.

23 National Cancer Institute, Division of Cancer Treatment and Diagnosis. Cancer therapy evaluation program. Adverse events/CTCAE. Available at: https://ctep.cancer.gov/protocolDevelopment/electronic_applications/ctc.htm\#ctc_40 (accessed December 23, 2018). 


\section{Liver Cancer}

24 Lencioni R, Llovet JM. Modified RECIST (mRECIST) assessment for hepatocellular carcinoma. Semin Liver Dis. 2010 Feb;30(1):52-60.

25 Eisenhauer EA, Therasse P, Bogaerts J, Schwartz LH, Sargent D, Ford R, et al. New response evaluation criteria in solid tumours: revised RECIST guideline (version 1.1). Eur J Cancer. 2009 Jan;45(2):228-47.

26 Kudo M, Matsui O, Izumi N, Kadoya M, Okusaka T, Miyayama S, et al.; Liver Cancer Study Group of Japan. Transarterial chemoembolization failure/refractoriness: JSH-LCSGJ criteria 2014 update. Oncology. 2014;87 Suppl $1: 22-31$.

27 Johnson PJ, Berhane S, Kagebayashi C, Satomura S, Teng M, Reeves HL, et al. Assessment of liver function in patients with hepatocellular carcinoma: a new evidence-based approach - the ALBI grade. J Clin Oncol. 2015 Feb;33(6):550-8.

28 Hiraoka A, Michitaka K, Kumada T, Izumi N, Kadoya M, Kokudo N, et al. Validation and Potential of AlbuminBilirubin Grade and Prognostication in a Nationwide Survey of 46,681 Hepatocellular Carcinoma Patients in Japan: The Need for a More Detailed Evaluation of Hepatic Function. Liver Cancer. 2017 Nov;6(4):325-36.

29 Harimoto N, Taguchi K, Shirabe K, Adachi E, Sakaguchi Y, Toh Y, et al. The significance of fibroblast growth factor receptor 2 expression in differentiation of hepatocellular carcinoma. Oncology. 2010;78(5-6):361-8.

30 Seo S, Hatano E, Higashi T, Hara T, Tada M, Tamaki N, et al. Fluorine-18 fluorodeoxyglucose positron emission tomography predicts tumor differentiation, P-glycoprotein expression, and outcome after resection in hepatocellular carcinoma. Clin Cancer Res. 2007 Jan;13(2 Pt 1):427-33.

31 Sung PS, Park HL, Yang K, Hwang S, Song MJ, Jang JW, et al. 18F-fluorodeoxyglucose uptake of hepatocellular carcinoma as a prognostic predictor in patients with sorafenib treatment. Eur J Nucl Med Mol Imaging. 2018 Mar;45(3):384-91.

32 Kodama K, Kawaoka T, Namba M, Uchikawa S, Ohya K, Morio K, et al. Correlation between Early Tumor Marker Response and Imaging Response in Patients with Advanced Hepatocellular Carcinoma Treated with Lenvatinib. Oncology. 2019;97(2):75-81.

33 Nishihara Y, Aishima S, Kuroda Y, Iguchi T, Taguchi K, Asayama Y, et al. Biliary phenotype of hepatocellular carcinoma after preoperative transcatheter arterial chemoembolization. J Gastroenterol Hepatol. 2008 Dec; 23(12):1860-8.

34 Buschauer S, Koch A, Wiggermann P, Müller M, Hellerbrand C. Hepatocellular carcinoma cells surviving doxorubicin treatment exhibit increased migratory potential and resistance to doxorubicin re-treatment in vitro. Oncol Lett. 2018 Apr;15(4):4635-40.

35 Yamashita Y, Matsukawa T, Arakawa A, Hatanaka Y, Urata J, Takahashi M. US-guided liver biopsy: predicting the effect of interventional treatment of hepatocellular carcinoma. Radiology. 1995 Sep;196(3):799-804. 